\title{
FGFR1 underlies obesity-associated progression of estrogen receptor-positive breast cancer after estrogen deprivation
}

Elizabeth A. Wellberg, ${ }^{1}$ Peter Kabos, ${ }^{2}$ Austin E. Gillen, ${ }^{3}$ Britta M. Jacobsen, ${ }^{1,4}$ Heather M. Brechbuhl, ${ }^{2}$ Stevi J. Johnson, ${ }^{1}$ Michael C. Rudolph, ${ }^{4}$ Susan M. Edgerton, ${ }^{1}$ Ann D. Thor, ${ }^{1}$ Steven M. Anderson, ${ }^{1}$ Anthony Elias, ${ }^{2}$ Xi Kathy Zhou, ${ }^{5}$ Neil M. Iyengar, ${ }^{6,7}$ Monica Morrow, ${ }^{8}$ Domenick J. Falcone, ${ }^{9}$ Omar El-Hely, ${ }^{7}$ Andrew J. Dannenberg, ${ }^{7}$ Carol A. Sartorius, ${ }^{1}$ and Paul S. MacLean ${ }^{4}$

'Department of Pathology and 'Division of Medical Oncology, Department of Medicine, University of Colorado Denver, Aurora, Colorado, USA. ${ }^{3}$ University of Colorado School of Medicine, RNA Bioscience Initiative, Aurora, Colorado, USA. ${ }^{4}$ Division of Endocrinology, Metabolism, \& Diabetes, Department of Medicine, University of Colorado Denver, Aurora, Colorado, USA. ${ }^{5}$ Department of Healthcare Policy and Research, Weill Cornell Medical College, New York, New York, USA. ${ }^{6}$ Department of Medicine, Memorial Sloan Kettering Cancer Center (MSKCC), New York, New York, USA. ${ }^{7}$ Department of Medicine, Weill Cornell Medical College, New York, New York, USA. ${ }^{8}$ Department of Surgery, MSKCC, New York, New York, USA. 'Department of Pathology and Laboratory Medicine, Weill Cornell Medical Center, New York, New York, USA.

Obesity increases breast cancer mortality by promoting resistance to therapy. Here, we identified regulatory pathways in estrogen receptor-positive (ER-positive) tumors that were shared between patients with obesity and those with resistance to neoadjuvant aromatase inhibition. Among these was fibroblast growth factor receptor 1 (FGFR1), a known mediator of endocrine therapy resistance. In a preclinical model with patient-derived ER-positive tumors, diet-induced obesity promoted a similar gene expression signature and sustained the growth of FGFR1-overexpressing tumors after estrogen deprivation. Tumor FGFR1 phosphorylation was elevated with obesity and predicted a shorter disease-free and disease-specific survival for patients treated with tamoxifen. In both human and mouse mammary adipose tissue, FGF1 ligand expression was associated with metabolic dysfunction, weight gain, and adipocyte hypertrophy, implicating the impaired response to a positive energy balance in growth factor production within the tumor niche. In conjunction with these studies, we describe a potentially novel graft-competent model that can be used with patient-derived tissue to elucidate factors specific to extrinsic (host) and intrinsic (tumor) tissue that are critical for obesity-associated tumor promotion. Taken together, we demonstrate that obesity and excess energy establish a tumor environment with features of endocrine therapy

License: This work is licensed unde the Creative Commons Attribution 4.0 International License. To view a copy of this license, visit http:// creativecommons.org/licenses/ by/4.0/.

Authorship note: EAW and PK contributed equally to this work. CAS and PSM contributed equally to this work.

Conflict of interest: The authors have declared that no conflict of interest exists.

Submitted: February 23, 2018

Accepted: June 19, 2018

Published: July 25, 2018

Reference information: JCI Insight. 2018;3(14):e120594. https://doi.org/10.1172/jci. insight.120594. resistance and identify a role for ligand-dependent FGFR1 signaling in obesity-associated breast cancer progression.

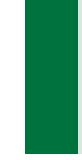

\section{Introduction}

The negative health effects of obesity extend to every organ system and include elevated risk and worse prognosis for most cancers. Breast cancer is the most frequently diagnosed cancer in women and is the second leading cause of cancer death. The majority of breast tumors $(>70 \%)$ express the estrogen receptor (ER) and are diagnosed after menopause. Women with obesity are more likely than their lean counterparts to face a poor breast cancer prognosis both before and after menopause (1-3). It is estimated that each 5 -unit increase in BMI translates to $\sim 8 \%$ greater risk of cancer recurrence or death (4). Furthermore, women with obesity frequently present with advanced disease, including larger tumors, higher tumor stage, and lymph node involvement $(4,5)$. Endocrine therapies, including selective ER modulators and aromatase inhibitors (AIs), are the standard treatment for most ER-positive breast cancers and act by blocking tumor ER activity or inhibiting adipose tissue aromatization of androgens to estrogens $(6,7)$. Unfortunately, nearly half of ER-positive tumors become endocrine-therapy resistant and recur, and this occurs with even greater frequency in the obese population. Although AIs effectively reduce circulating estrogens by $\sim 95 \%$ regardless of adiposity level (8), women with obesity are less likely to benefit $(9,10)$. 
Various mechanisms are responsible for either acquired or de novo resistance to endocrine therapy, including mutations in ESR1, amplification and overexpression of growth factor receptors (GFRs), and alterations in cell cycle machinery (11). Activated growth factor receptor pathways, such as human epidermal growth factor receptor-2 (HER2), insulin-like growth factor 1 receptor (IGF1R), and fibroblast GFR 1 (FGFR1), converge on the PI3K/Akt/mTOR and Raf/Mek/Erk pathways to support tumor cell proliferation and survival after estrogen deprivation and serve as current targets of approved therapies for steroid hormone receptor-positive disease. It is currently unclear whether obesity impacts GFR expression and activation to influence breast cancer endocrine therapy resistance. A recent report showed that the prevalence of obesity in women has increased to $40 \%$ (12). Despite these observations, obesity is not frequently included as a biological variable in preclinical breast cancer studies. One of the likely reasons for this limitation is the difficulty in promoting obesity in transplant-competent mouse strains that can sustain ER-positive tumors.

To study the relationship between obesity and endocrine therapy resistance, we investigated similarities in primary tumors between women with elevated BMI $\left(\geq 25 \mathrm{~kg} / \mathrm{m}^{2}\right)$ and women who were treated with neoadjuvant aromatase inhibition, with emphasis on GFR pathways. We then developed a graft-competent mouse model of obesity to utilize the emerging breast cancer patient-derived xenograft (PDX) resources (13). Here, we describe a method to promote obesity in immune-compromised mice that takes advantage of the high penetrance of diet-induced obesity in the C57BL/6 background, the immune deficiency of the Rag1-null model, and optimal housing and nutrient conditions for achieving maximal adiposity in mature female mice. By investigating well-characterized and potentially novel ER-positive breast cancer models in the context of obesity, we found that breast cancers showed divergent responses to estrogen deprivation. Tumors that did not respond to treatment in the obese mice displayed evidence of ligand-dependent FGFR1 activation, which is a mediator of endocrine therapy resistance $(14,15)$. In primary human breast tumors, high levels of pFGFR1 associated with elevated BMI $\left(\geq 25 \mathrm{~kg} / \mathrm{m}^{2}\right)$ and also with shorter disease-free and breast cancer-specific survival. We also identified estrogen withdrawal-induced (EWD-induced) weight gain as a driver of FGF1 production by mammary adipose tissue, implicating the obese host environment in breast cancer progression through GFR pathway activation. Our model has applicability to a variety of cancer types in which obesity is a negative prognostic factor. Moreover, our study suggests that obesity should be considered a unique variable in clinical breast cancer treatment.

\section{Results}

Obesity promotes activation of pathways that associate with endocrine therapy resistance. To determine the relationship between obesity-associated breast cancer and the response of ER-positive breast tumors to endocrine therapy, we compared previously published tumor gene expression data from patients with elevated BMI $\left(\geq 25 \mathrm{~kg} / \mathrm{m}^{2}\right)(16)$ to that from patients whose tumors responded or did not respond to short-term letrozole treatment $(17,18)$. Large-scale studies have shown little overlap in gene signatures across breast tumor datasets that predict response to therapy $(19,20)$; therefore, we analyzed the putative upstream regulators of gene expression profiles to understand the potential extrinsic factors influencing tumor behavior. Using this approach, we identified activated GFR and kinase signaling pathways that were common to tumors from patients with a high BMI and either responders or nonresponders to letrozole (Figure 1A). Among the putatively activated pathways that were common to both obesity and a poor response to letrozole were IGF1R and FGFR1, which have been implicated in endocrine therapy resistance $(14,15,21)$. Of the 5 upstream regulatory pathways shared by tumors from overweight patients and nonresponders, FGFR1 and, to a lesser extent, IGFR1 were predicted to be decreased in tumors that did respond to letrozole (Figure 1B). To validate the association between activated FGFR1 and endocrine therapy response, we examined levels of phosphorylated FGFR1 (pFGFR1; Figure 1C) in primary breast tumors from women treated with tamoxifen and found that high levels of pFGFR1 ( $\geq 75 \%$ positive cells) associated with a shorter disease-free and disease-specific (overall) survival compared with low level expression (Figure 1D). Together, these data suggest that activation of FGFR1 signaling may drive a gene expression program shared between obesity-associated breast cancer and endocrine therapy resistance.

Thermoneutral housing and high fat/high sucrose promote obesity in female Rag1-null mice. To investigate the mechanisms by which obesity promotes breast cancer progression, we developed a diet-induced obese murine model in which to grow breast cancer PDX. Although diet-induced and transgenic mouse models of obesity are routinely used, it has been challenging to develop an obese graft-competent model for breast cancer studies. 
A
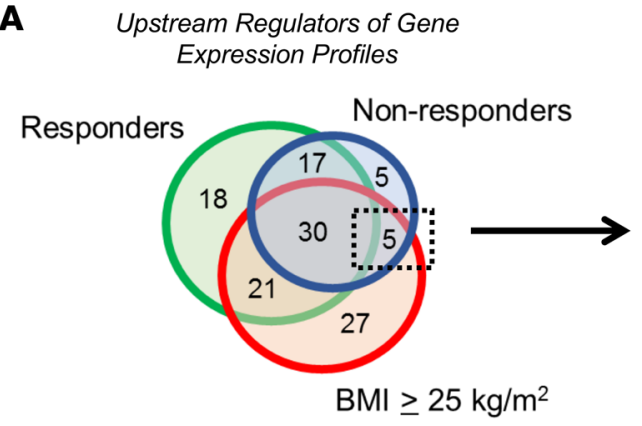

B Upstream Regulators Common to

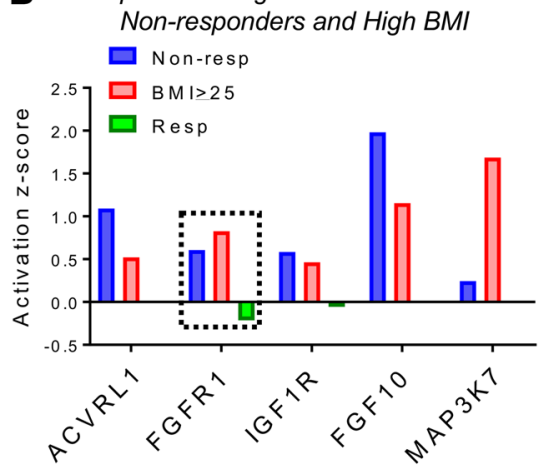

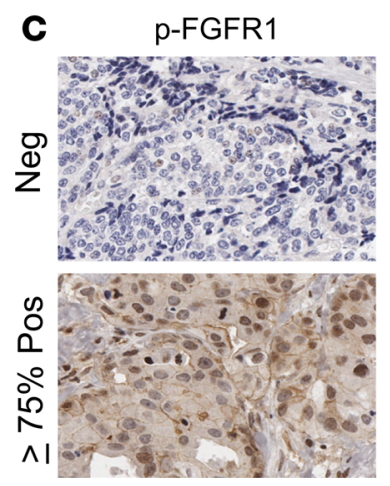
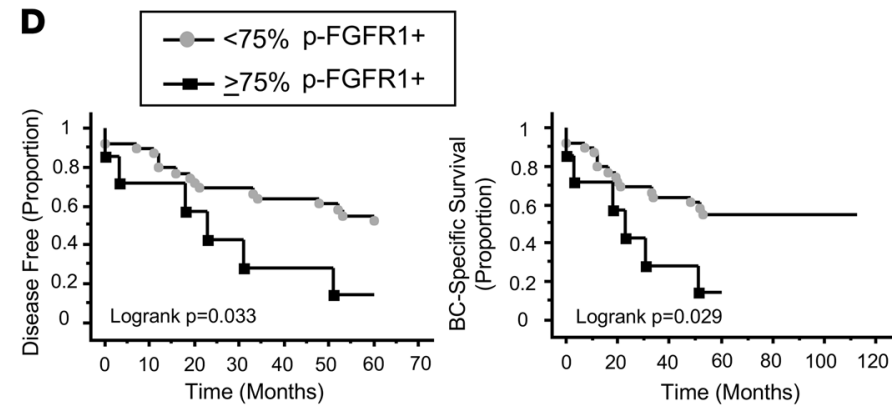

Figure 1. FGFR1 activation associates with obesity and a poor response to endocrine therapy. (A) Venn diagram of unique and shared activated regulatory pathways between tumors from those who did not respond to letrozole (Nonresponders, blue, $n=57$ pathways), those who did respond (Responders, green, $n=86$ pathways), and estrogen receptor-positive (ER-positive) tumors from women with elevated BMI (red, $n=83$ pathways). (B) $Z$ scores of activated upstream regulators reported by Ingenuity to be common to nonresponders and patients with elevated BMI $(P<0.05)$. (C) Representative images of primary human breast tumors stained for pFGFR1. Top panel, negative tumor ( $0 \%$ positive); bottom panel, $>75 \%$ positive. Magnification, 20×. (D) Kaplan Meier survival curves showing disease-free (left) and breast cancer-specific (right) survival in patients based on pFGFR1 staining. $n=39,<75 \%$ positive; $n=7, \geq 75 \%$ positive.

To overcome these challenges, we took advantage of the tendency for diet-induced obesity in the C57BL/6 mouse strain $(22,23)$, the reduced immune function of the Rag1-null mutation (24), and thermoneutral housing conditions, which support obesity development in immune-compromised mice (25). Cages placed on warming blankets set at $40^{\circ} \mathrm{C}$ resulted in internal cage temperatures of $\sim 30^{\circ} \mathrm{C}$, which is within the mouse thermoneutral zone (26) (Figure 2A, red box). In contrast, cages housed at room temperature remained at $\sim 23^{\circ} \mathrm{C}$ (Figure 2A, control). Body weights were measured in all mice beginning at 6 weeks of age (Figure 2B). The introduction of thermoneutral housing (Figure 2B, bold arrow) preferentially led to an accelerated weight gain in mice on the high-fat/high-sucrose (HFHS) diet. Mice on the low-fat/low-sucrose (LFLS) diet continued on a similar weight-gain trajectory, despite the same thermoneutral temperatures. Lean mass was increased in HFHS-fed compared with LFLS-fed mice (Figure 2C); however, the accelerated weight gain induced by warming led to higher fat accumulation (Figure 2D), which was significantly greater in HFHS-fed compared with LFLS-fed mice after 6 weeks of thermoneutral housing (Figure 2D). Although the C57BL/6 strain is inbred (isogenic), we observed a distribution in the range of body fat percentage across mice fed HFHS diets (Figure 2D), potentially due to pre- and/or postnatal programming effects of obesity susceptibility (27-29). We performed an equivalent study using NOD-Scid-I12r-null (NSG) mice, which are commonly used as recipients for human PDX tumors (30). NSG mice were fed LFLS or HFHS diets and housed at thermoneutral temperatures. Although body weight increased modestly with HFHS feeding and HFHS-fed NSG mice were heavier than those given the LFLS diet (Supplemental Figure 1; supplemental material available online with this article; https://doi.org/10.1172/jci.insight.120594DS1), the level of adiposity was not significantly different between the 2 groups (Supplemental Figure 1).

After determining that the Rag1-null mice were obesity prone, we performed all subsequent tumor studies using this mouse strain and ER-positive breast cancer lines. To demonstrate the utility of the model, mice were 
A

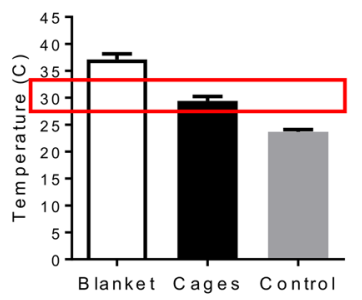

$\mathbf{F}$

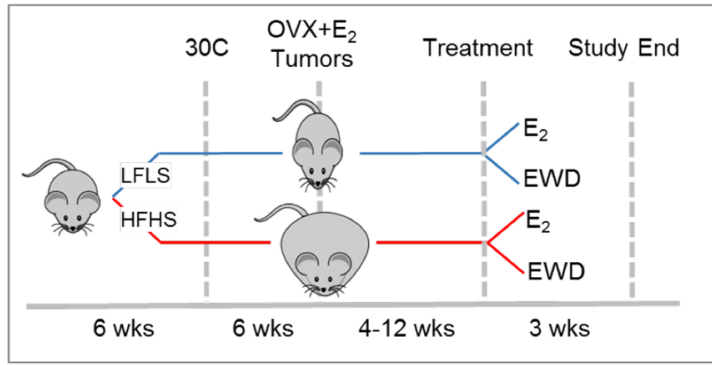

B

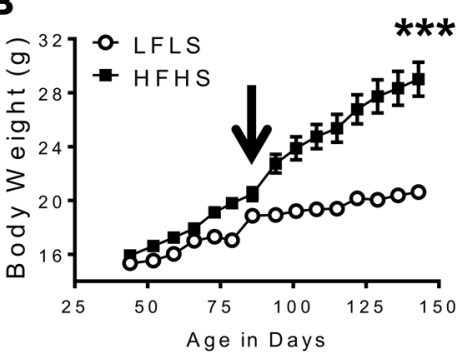

G

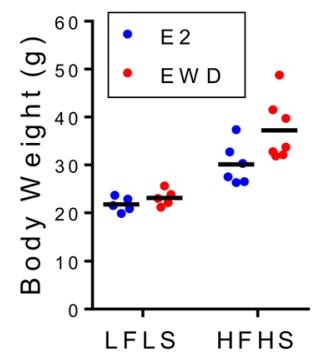

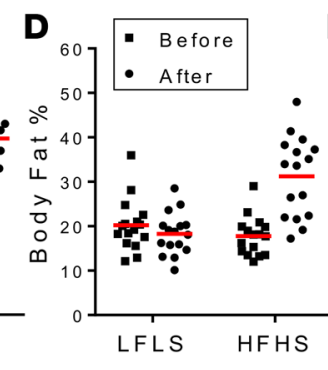

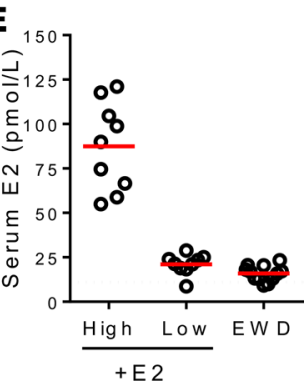

H

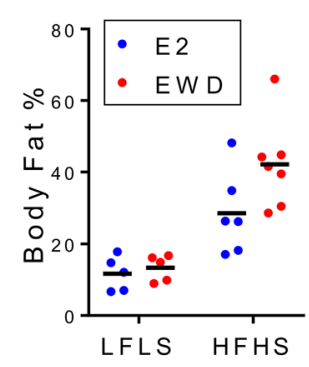

I

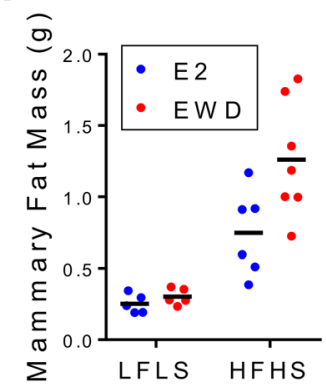

J

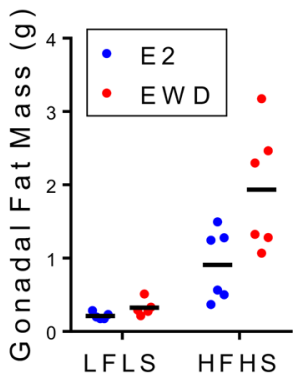

K

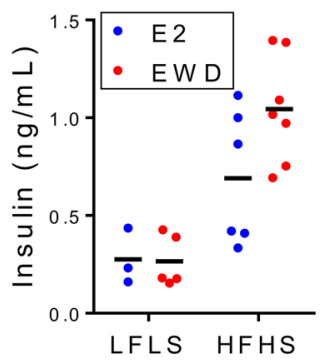

$\mathbf{L}$

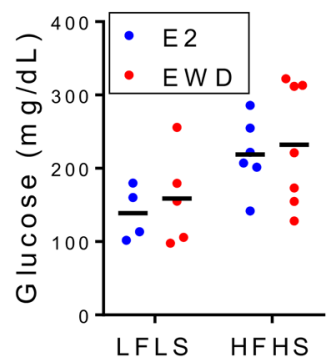

M

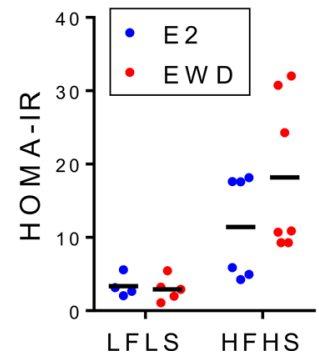

$\mathbf{N}$

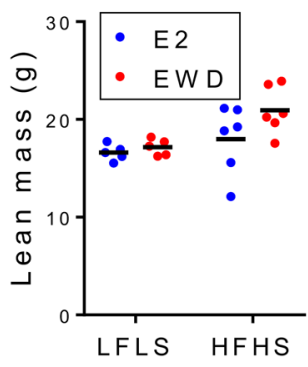

Figure 2. Development of obesity in Rag1-null mice. (A) Surface temperature of warming blanket (open bar), internal temperature of cages housed on blankets (black bar), and cages housed at control room temperatures (gray bar). $n=3$. Red box indicates mouse thermoneutral temperature zone. (B) Body weight of LFLS (lean) and HFHS (obese) fed Rag1-null mice. $n=15$ lean, 16 obese. Arrow indicates start of thermoneutral housing ( ${ }^{* * *} P<0.001$, unpaired $t$ test). (C) Lean mass before and 6 weeks after warming. Adiposity effect $P<0.0001$, warming effect $P=0.0008$, interaction $P=0.4$. $n=16$ mice per group. (D) Percent body fat before and 6 weeks after warming. Adiposity effect $P=0.0014$, warming effect $P=0.0005$, interaction $P<0.0001$. $n=16$ mice per group. (E) Diagram of Rag1-null tumor studies. OVX, E2 supplementation, and tumor implantation are performed on mature lean and obese mice. Estrogen withdrawal (EWD) is used as treatment, with maintenance on E2 as a control. (F) Serum E2 levels measured by ELISA in OVX female mice supplemented with $1 \mathrm{mg}$ (high) or 0.25 mg (low) E2 pellets or after EWD. Dashed line indicates lowest limit of detection. Means denoted by red bars. (G-N) Effects of EWD or E2 maintenance on (G) body weight (adiposity $P<0.0001$, treatment $P=0.03$, interaction $P=0.13$ ), (H) body fat (adiposity $P=0.0001$, treatment $P=0.07$, interaction $P=0.15$ ), (I) mammary fat mass (adiposity $P<0.0001$, treatment $P=0.03$, interaction $P=0.06$ ), (J) gonadal fat mass (adiposity $P<0.0001$, treatment $P=0.02$, interaction $P=0.05$ ), (K) serum insulin (adiposity $P=0.0001$, treatment $P=0.07$, interaction $P=0.14$ ), (L) serum glucose (adiposity $P=0.01$, treatment $P=0.56$, interaction $P=0.9$ ), (M) HOMA-IR (adiposity $P=0.002$, treatment $P=0.3$, interaction $P=0.3$ ), and (N) lean mass (adiposity $P=0.45$, treatment $P=0.76$, interaction $P$ $=0.99$ ). Data were analyzed by 2-way ANOVA with test for interaction. $n=5$ lean E2, $n=5$ lean EWD, $n=6$ obese E2, $n=7$ obese EWD.

ovariectomized (OVX) and supplemented with estradiol (E2) to achieve serum levels that approximate the follicular phase in premenopausal women (Figure 2E, high, $1 \mathrm{mg}$ E2/pellet; ref. 31), postmenopausal (Figure 2E, low, $0.25 \mathrm{mg}$ E2/pellet; ref. 8) conditions, or postendocrine therapy (Figure 2E, EWD). Here, each tumor study followed a similar design (Figure 2F), where juvenile female Rag1-null mice were matured to either lean or obese adults on LFLS or HFHS diets, respectively. At maturity, mice were OVX, implanted with $0.25 \mathrm{mg}$ E2 pellets, and breast cancer cells or PDX tumor fragments were injected orthotopically in the inguinal mammary fat pads. Once tumors were established, we either performed EWD treatment to mimic estrogen deprivation by AIs or mice were maintained on supplemental E2 for the duration of the study, which lasted 3 additional weeks.

Diet-induced obesity influences the metabolic response to EWD. Estrogen deprivation therapy and the menopausal transition each impact metabolic function and adipose accumulation in women (32-35). To monitor these changes, we measured several parameters in LFLS- and HFHS-fed Rag1-null mice that 
were either maintained on E2 or that underwent EWD treatment. There was a significant effect of diet on body weight (Figure 2G), body fat (Figure 2H), mammary (Figure 2I) and gonadal (Figure 2J) fat masses, serum insulin (Figure $2 \mathrm{~K}$ ) and glucose (Figure 2L), and the homeostatic model assessment of insulin resistance (HOMA-IR), a measure of insulin sensitivity (Figure 2M). EWD treatment significantly increased body weight, as well as mammary and gonadal fat masses. By pairwise comparison, gonadal and mammary fat masses significantly increased with EWD only in HFHS-fed mice. Lean mass was unaffected by diet or treatment (Figure $2 \mathrm{~N}$ ). Overall, the negative metabolic effects of EWD were worse in HFHS compared with LFLS Rag1-null mice.

Distinct effects of obesity on ER-positive tumor growth. We have previously shown in a rat model of chemically induced mammary cancer that diet-induced obesity promotes the survival and growth of ER-positive tumors after the loss of ovarian estrogen production (36-38). To extend our work, we implanted MCF7 cells or a potentially novel ER-positive PDX (UCD12; ref. 39) into mature, OVX female Rag1-null mice that were supplemented with low-dose E2. Tumor growth in the presence of E2 was greater in HFHS versus LFLS mice (Figure 3, A and C). Both MCF7 and UCD12 tumors exhibited a variable response to EWD in LFLS and HFHS mice (Figure 3, B and D). Similar to MCF7 tumors, some UCD12 tumors in LFLS mice continued to grow, while others regressed after EWD (Figure 3D, left). In contrast, all UCD12 tumors in HFHS mice continued to grow after EWD (Figure 3D, right). Overall, in the presence of E2, obesity potentiated the growth of ER-positive MCF7 and UCD12 tumors; however, excess adiposity only sustained the growth of UCD12 tumors after EWD. In this regard, these tumor types model the responders (MCF7) and nonresponders (UCD12) to estrogen deprivation therapy in the context of obesity.

The prevailing explanation for the obesity-breast cancer link centers on excess estrogen production; however, progesterone receptor (PR), which is commonly used as an indicator of estrogenic activity, was undetectable after EWD in either MCF7 or UCD12 tumors (Figure 3, E and F). Similarly, there was no effect of obesity on UCD12 tumor expression of well-known ER target genes Greb1 and Tff1 after EWD (Figure 3G). In Rag1-null mice, mammary adipose $\mathrm{E} 2$ was below detection (Figure $3 \mathrm{H}$ ), and differences were not found in circulating E2 between LFLS- and HFHS-fed mice (13.9 \pm 3.7 pM vs. $10.5 \pm 2.1 \mathrm{pM}$, respectively). This is consistent with our previous report in female rats after OVX, where plasma levels did not differ with obesity and adipose tissue levels were below detection limits, simulating an environment of aromatase inhibition (38); however, levels of estrogen below detectable amounts may still sufficiently activate ER in cancer cells (40).

FGFR1 associates with obesity-associated tumor growth after estrogen loss. The lack of elevated tumor ER target gene expression with obesity and the low levels of E2 in the adipose tissue of mice indicated that estrogen-independent mechanisms may be responsible for UCD12 tumor progression after EWD. We performed RNA-sequencing analysis of tumors from LFLS and HFHS mice, with and without E2 supplementation. Overall, 785 genes were differentially expressed across all 4 groups (FDR $\leq 0.01 ;$ Abs $\log$ FC = 1; Figure 4A). In LFLS mice, 355 genes increased following EWD, while 430 genes decreased (Figure 4A). Many of the genes that were decreased by EWD in LFLS mice were elevated in HFHS mice, regardless of EWD treatment, and vice versa (Figure 4A). We characterized the pathways and functions associated with obesity after EWD using Ingenuity Pathway Analysis. Networks involving apoptosis, necrosis, and cell death were all predicted to be inactive in tumors from HFHS versus LFLS, while metastasis, invasion, and migration of cancer cells were predicted to be active (Figure 4B). Among the differentially affected pathways was FGF/FGFR1 signaling, which was putatively active in the context of obesity after EWD. We confirmed overexpression of FGFR1 in UCD12 compared with MCF7 cells (Figure 4C and Supplemental Figure 2), suggesting signaling through this pathway may be responsible for the differential tumor progression. Furthermore, pFGFR1 was elevated in tumor lysates from HFHS versus LFLS mice after EWD in an unbiased phospho-receptor tyrosine kinase array screen (Supplemental Figure 3). Among the genes elevated in tumors from EWD-treated HFHS mice were those encoding extracellular matrix remodeling enzymes, such as matrix metalloproteinases 1 and 3 and hyaluronic acid synthase 2 (Figure 4D, left), as well as structural proteins, including collagen $1 \mathrm{~A} 2$ and fibronectin 1 (Figure $4 \mathrm{D}$, right). Consistent with a recent report (41), analysis of trichrome-stained tumors suggested increased collagen deposition in HFHSfed mice compared with LFLS-fed mice after EWD (Figure 4E), raising the possibility that obesity may influence tumor progression through modulation of the extracellular matrix.

FGFR1 phosphorylation associates with poor patient outcomes and with obesity. FGFR1 is amplified and/ or overexpressed in 15\%-20\% of ER-positive breast cancers and supports endocrine therapy resistance $(15,42)$. Compared with UCD12 PDX tumors from LFLS mice, those from HFHS mice had higher 
A

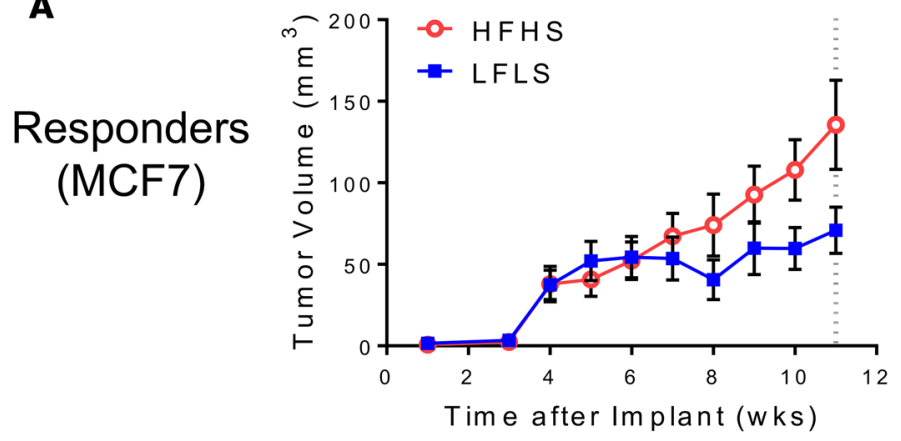

C

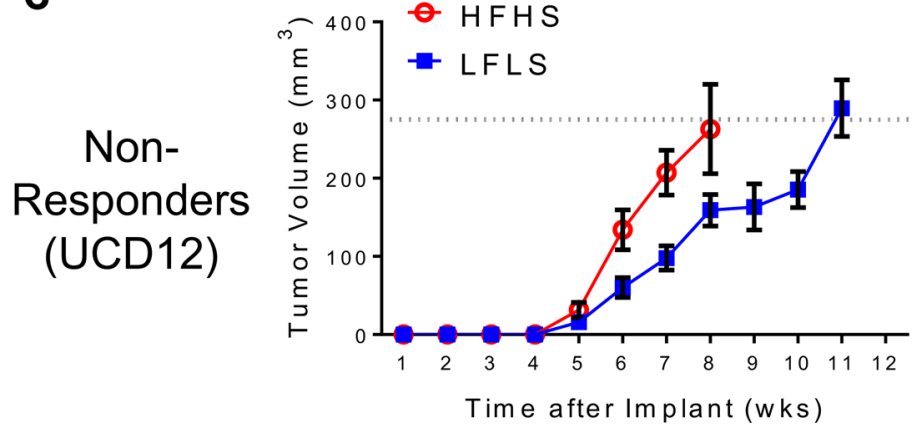

E
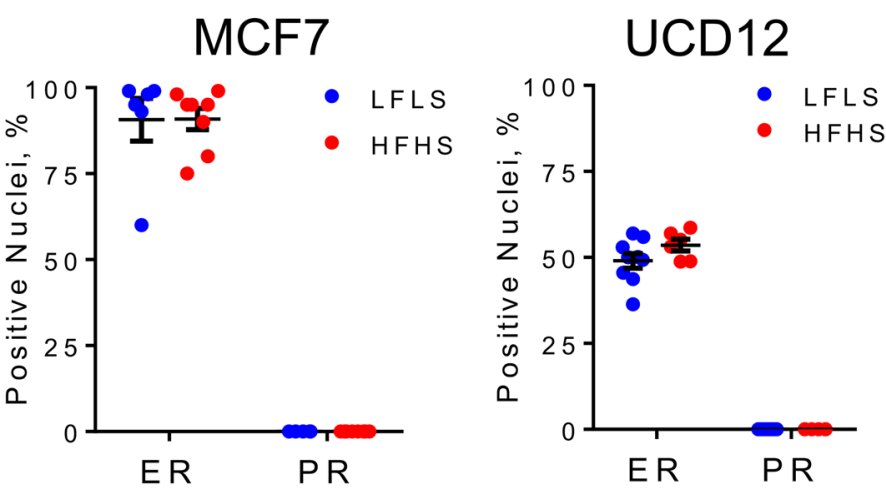

B
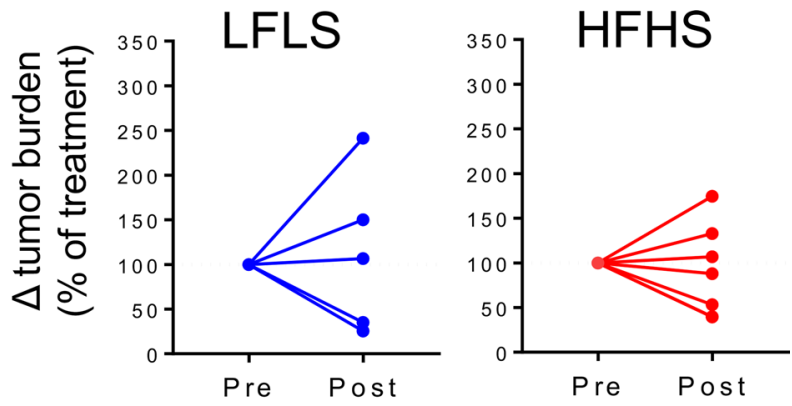

D
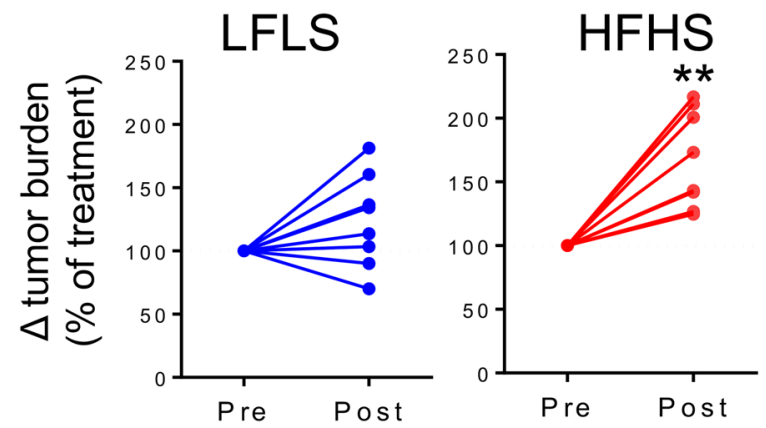

$\mathbf{F}$

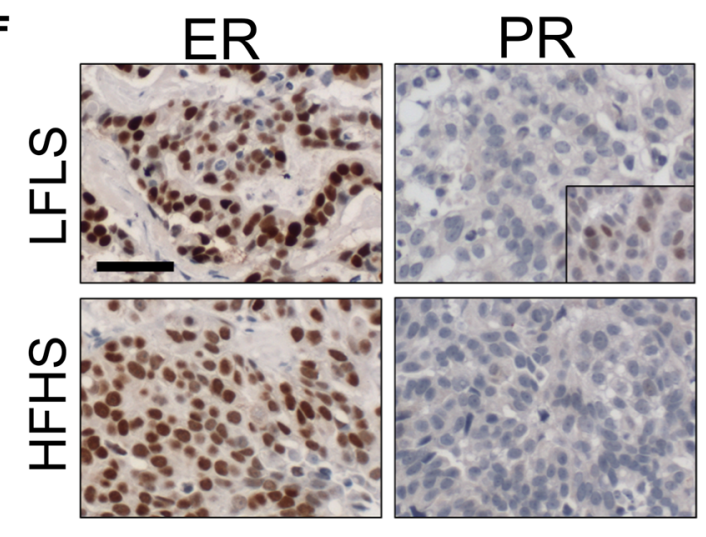

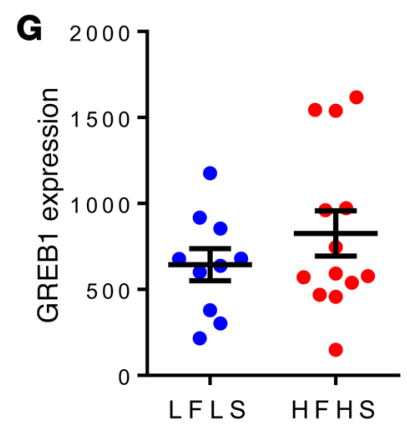
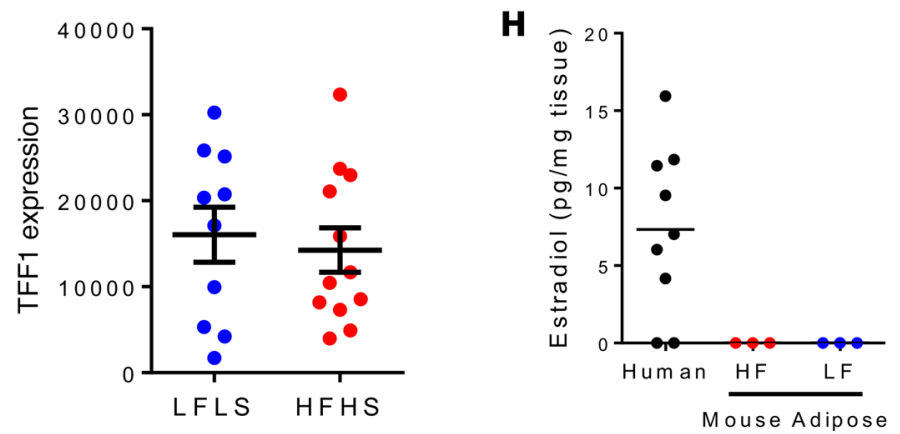

Figure 3. Responders and nonresponders to EWD in the context of obesity. (A) Total tumor volume in lean $(n=9)$ and obese $(n=12)$ E2-treated mice. Dashed line indicates tumor size at treatment. (B) Change in tumor volume in lean (left) and obese (right) mice from the time of EWD treatment (Pre) until 3 weeks after (Post), represented as a percent of tumor volume at treatment. (C) UCD12 PDX tumor volume in lean ( $n=17)$ and obese $(n=16)$ E2-treated mice. Dashed line indicates tumor size at treatment. (D) Tumor burden per mouse prior to (Pre) and after (Post) EWD treatment, in obese $(n=8)$ and lean $(n=8)$ mice. Data calculated as percent change from treatment volume. ${ }^{* *} P=0.002$. Paired $t$ test. (E) Percent ER- and PR-positive nuclei in post-EWD MCF7 and UCD12 tumors. $n=7$ tumors per group. (F) Representative images of ER and PR IHC from MCF7 tumors. Inset shows PR-positive control slide (E2-treated tumor). Scale bar: $100 \mu \mathrm{m}$. (G) Expression of GREB1 and TFF1 in post-EWD tumors. (H) Tissue estradiol levels from human breast or mouse mammary adipose. 
A
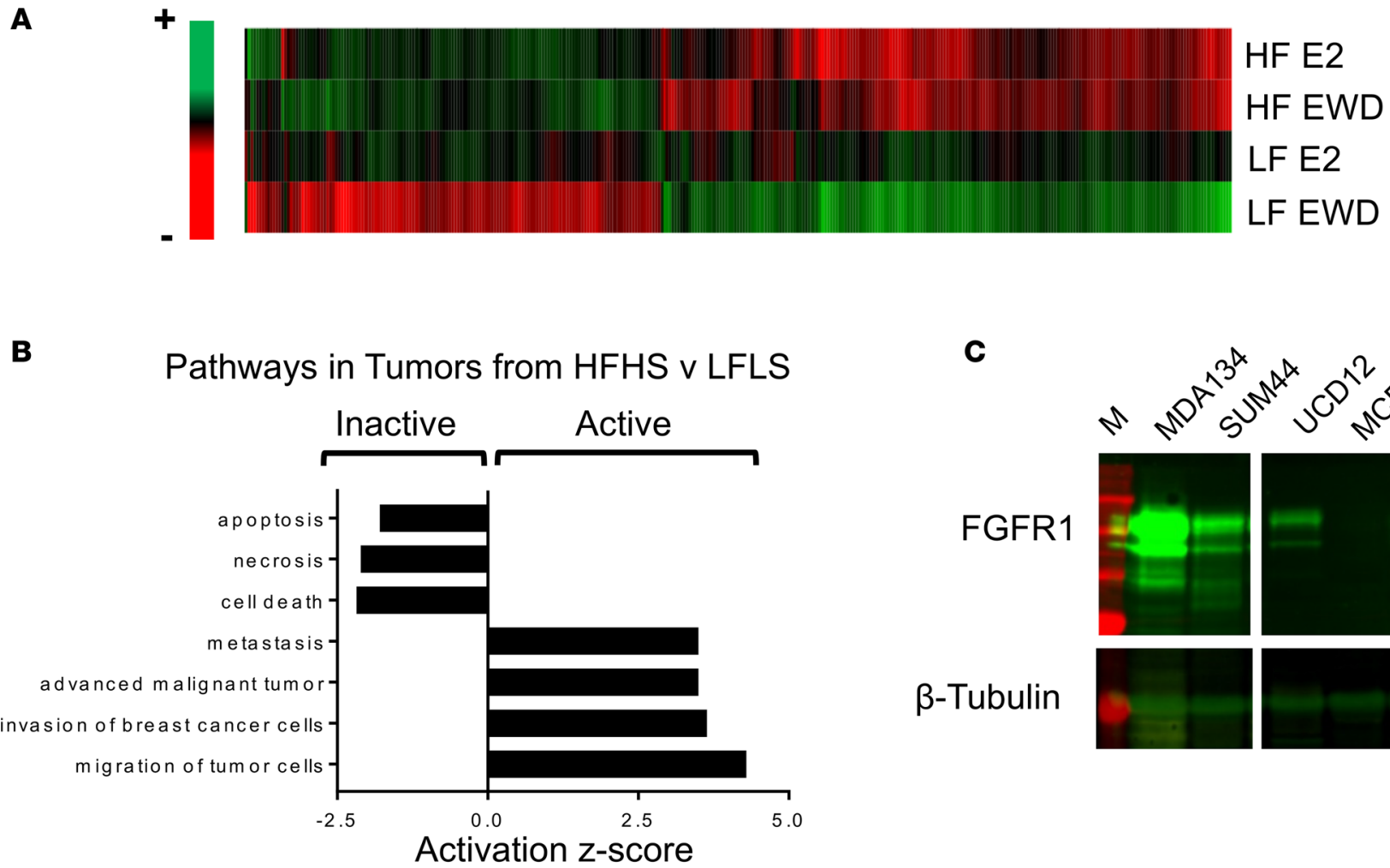

C

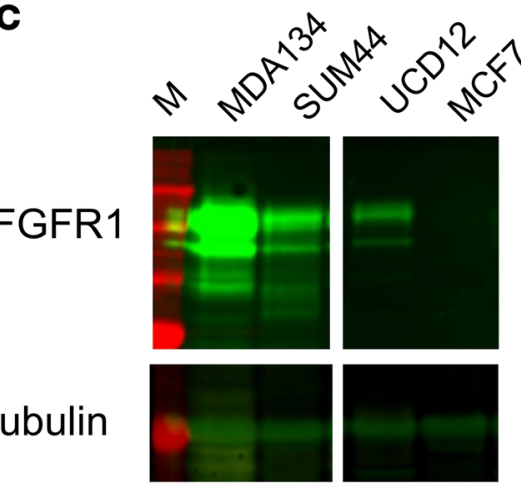

D

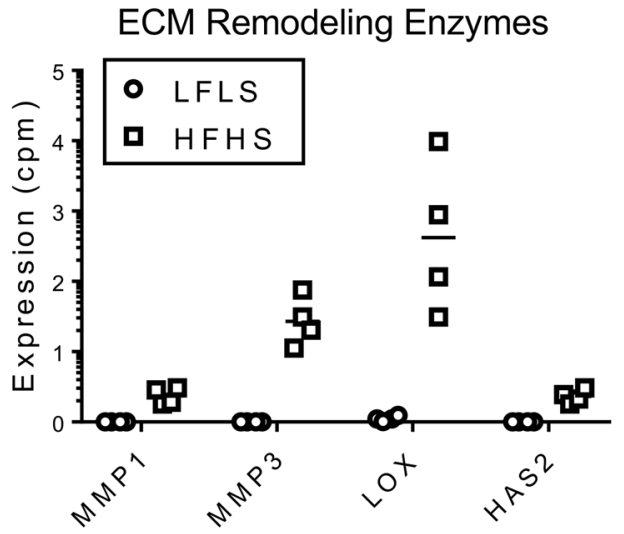

ECM Proteins

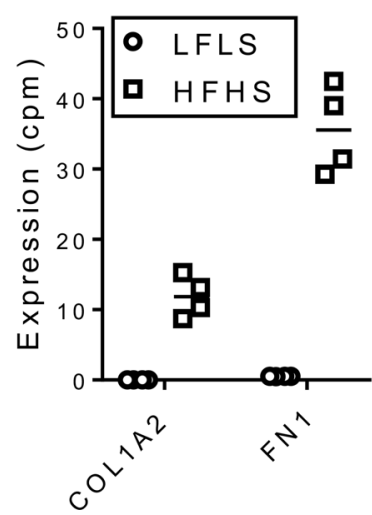

E

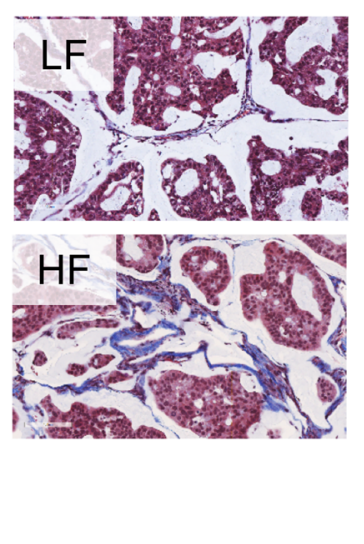

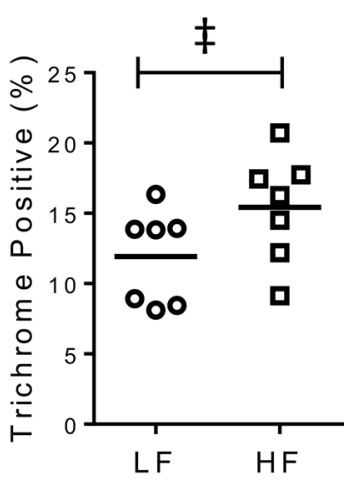

Figure 4. Obesity promotes endocrine therapy resistance phenotype after EWD. (A) Heatmap of differentially expressed genes in tumors ( $n=4$ per group) from lean or obese E2- or EWD-treated mice. FDR $<0.01$, absolute log fold change $=1$. (B) Pathways predicted to be active or inactive in the obese based on gene expression profiles. All $P<0.05$. (C) Immunoblot of FGFR1 and $\beta$-tubulin in control (MDA134 and SUM44) cells and UCD12 and MCF7 tumors. (D) Expression of ECM remodeling enzymes (left) and structural proteins (right) in tumors from lean and obese mice after EWD. (E) Representative images of trichrome staining in tumors from lean and obese mice after EWD and quantification with Aperio Digital Pathology System (right panel); magnification, $10 \times .{ }^{\ddagger} P<0.1$.

levels of pFGFR1 (Figure 5, A and B) with similar levels of total FGFR1 (Figure 5B). This difference was confirmed in a separate study using a cell line derived from the UCD12 tumor, referred to as PT12, injected into the inguinal mammary gland (Figure 5, C and D), indicating that this characteristic of the tumor persisted after multiple passages in vitro. We further investigated the relevance of FGFR-driven endocrine resistance in the context of obesity in a cohort of postmenopausal women with newly diagnosed ER-positive/HER2-negative breast cancers who were preoperatively treated with 4 months of neoadjuvant exemestane. In these postmenopausal women treated with AI, pFGFR1 - but not total FGFR1 - was significantly higher in tumors from women who were overweight or obese (BMI $\geq 25$ $\mathrm{kg} / \mathrm{m}^{2}$ ) compared with those that were lean (BMI $<25$; Figure $5, \mathrm{E}$ and $\mathrm{F}$ ). To further elucidate the respective contributions of FGFR and ER signaling to tumor progression in this model, HFHS-fed mice 
bearing UCD12 PDX tumors were treated with EWD and either the FGFR inhibitor BGJ398 or the AI letrozole. Compared with control tumors, which continued to grow after EWD, tumors from those treated with BGJ398 regressed after EWD treatment (Figure 5G). In Rag1-null females, the UCD12 PDX tumors continued to grow after EWD in the presence of letrozole (Figure 5G), consistent with evidence suggesting estrogen-independent tumor progression (Figure 3).

Weight gain in the obese supports FGF1 ligand production by adipose tissue. FGFR signaling can be activated through ligand-independent (e.g., receptor amplification or activating mutation) or ligand-dependent (e.g., paracrine, autocrine, or endocrine FGF production) mechanisms. The lack of differences in total FGFR1 levels between tumors from LFLS and HFHS mice suggested that the obese environment was not selecting for a tumor cell population with FGFR1 amplification. Instead, this observation indicated a possible influence of obesity on ligand-dependent signaling. To investigate this, we analyzed expression of FGF ligands within the tumors and in mammary adipose tissue adjacent to the tumors. In adipose tissue, FGF1 and FGF2 in particular act through paracrine or autocrine mechanisms to initiate FGFR1 signaling, are produced during adipose tissue expansion (weight gain), and are elevated with obesity $(43,44)$. FGF2 expression was not different between LFLS and HFHS mice (Supplemental Figure 4A); however, FGF1 expression was significantly increased in mammary adipose tissue after EWD only in HFHS mice (Figure 6A). EWD promoted weight gain (Figure 6B) and expansion of mammary adipocytes (Figure 6, C and D), with the largest adipocytes seen in HFHS mice (Figure 6D). FGF1 expression directly correlated with the rate of weight gain following EWD (Figure 6E, left) and mammary fat mass (Figure $6 \mathrm{E}$, middle), and with the average adipocyte diameter (Figure 6E, right). A previously published study was analyzed to determine whether FGF1 expression associated with weight gain in human adipose tissue (45). In paired biopsies of s.c. adipose tissue collected from obese subjects before and after intentional weight gain, FGF1 expression increased significantly only in those obese individuals that were classified as metabolically abnormal (Figure 6F; unhealthy) by intrahepatic triglyceride content and insulin sensitivity (45). This indicates an impaired response to a positive energy balance in adipose tissue growth factor production. Finally, in human breast adipose tissue, FGF1 expression was significantly elevated in patients with $\mathrm{BMI} \geq 30 \mathrm{~kg} / \mathrm{m}^{2}$ compared with $<25 \mathrm{~kg} / \mathrm{m}^{2}$ (Figure $6 \mathrm{G}$, left; Supplemental Table 1) and correlated with BMI (Figure 6G, middle), as well as breast adipocyte diameter (Figure 6G, right). The effect of obesity on FGF1 expression appears to be specific to adipose tissues, as tumor levels of FGF1 and FGF2 did not differ between treatment groups (Supplemental Figure 4, B and C). Taken together, these results suggest that a positive energy balance (i.e., weight gain) in the context of obesity and/or metabolic dysfunction promotes FGF ligand production by adipose tissue, which may activate receptors in nearby breast cancer cells to promote growth after estrogen deprivation (Figure 7).

\section{Discussion}

This study is the first to our knowledge to leverage a diet-induced obese graft competent model with human tumors and a series of clinical studies to reveal how obesity-related extrinsic and intrinsic factors converge to promote ER-positive breast tumor growth in the absence of estrogen. These experiments are particularly relevant to the important clinical problem of endocrine therapy resistance, which occurs with greater frequency in women with obesity $(3,5)$. Our observations present a scenario in which overnutrition under isothermic conditions increases the expression of FGF1 in adipose tissues in women with obesity and/or metabolic disease. In this environment, breast tumors expressing FGFR1 would be particularly susceptible to FGF ligand-dependent survival and growth after estrogen deprivation. Consistent with this scenario, amplification and overexpression of FGFR1 are reported to occur in up to $20 \%$ of luminal (ER-positive) subtype breast cancers (15) and are implicated in resistance to endocrine therapy $(14,15,46)$. Recently, FGFR1 was reported to interact with ER in the cytoplasm and nucleus of endocrine-resistant breast cancer cells, suggesting that ER remains functional through growth factor pathway activation, despite estrogen deprivation (14). Taken together, these observations support a dual-requirement hypothesis that we have previously presented $(38,47)$, in which metabolic impairment associated with obesity converges with a positive energy balance following estrogen deprivation to form a tumor-promoting environment (Figure 7). To tackle this problem clinically, it may only be necessary to improve metabolic function, either pharmacologically or through exercise, or to prevent EWD-induced weight gain. In theory, either of these approaches should sufficiently inhibit the development of endocrine therapy resistance observed in women with obesity. In addition, FGFR inhibitors may be uniquely beneficial in combination with aromatase inhibition as a first-line therapy in those with elevated BMI. 
A PDX

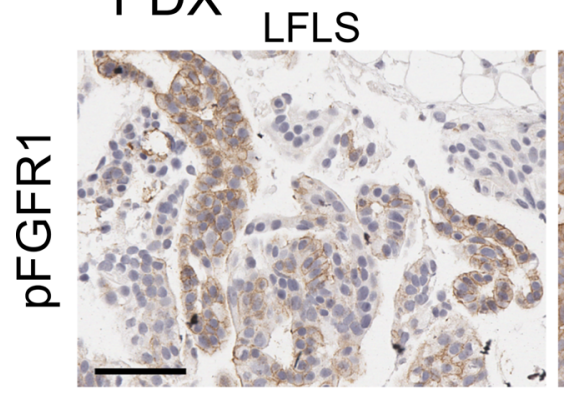

HFHS

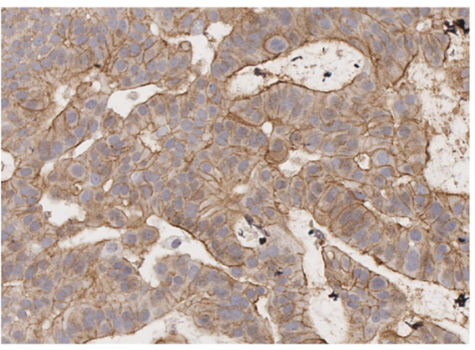

B
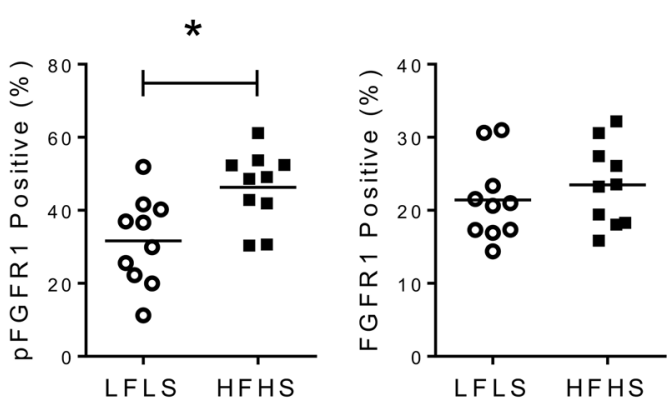

c Breast Cancer Cell Line

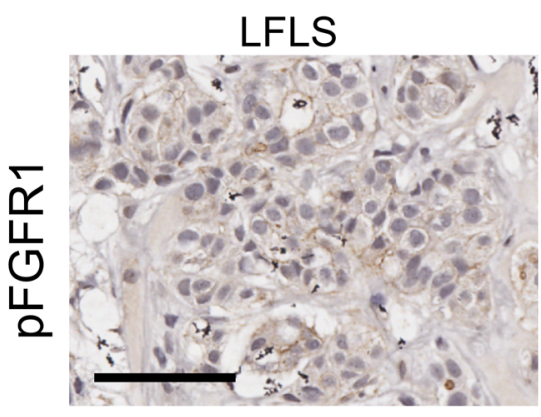

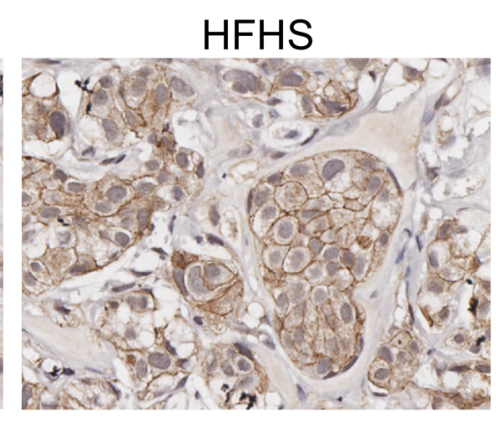

HFHS

E Primary Human Tumor

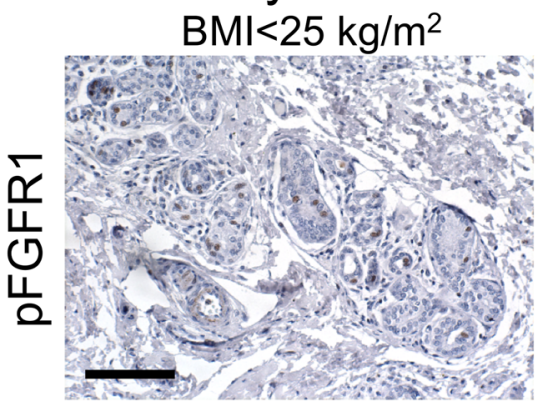

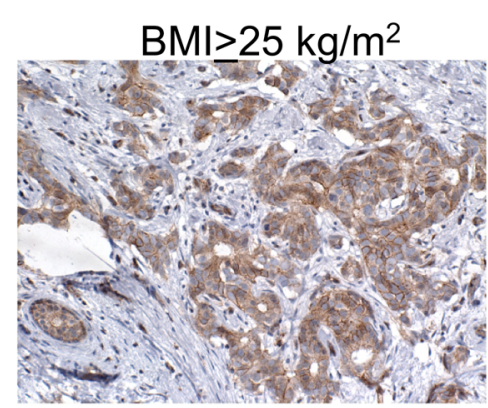

D

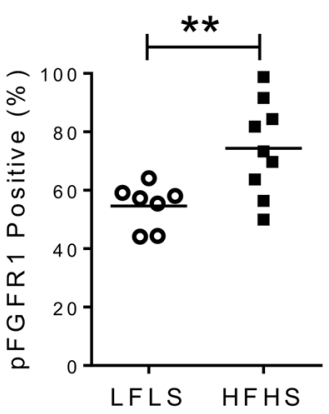

$\mathbf{F}$
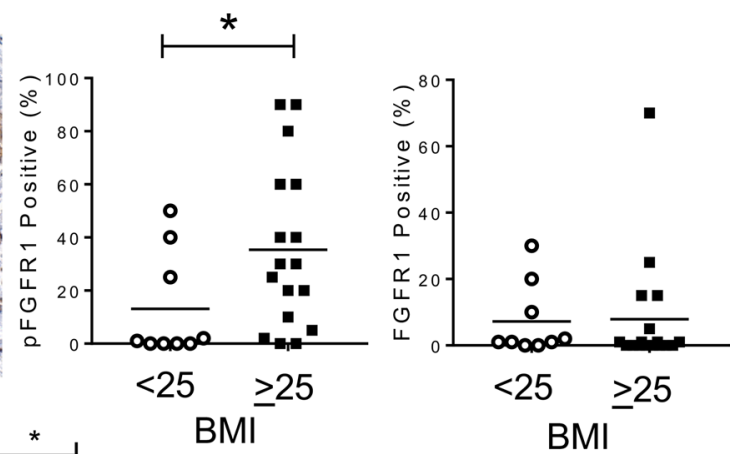

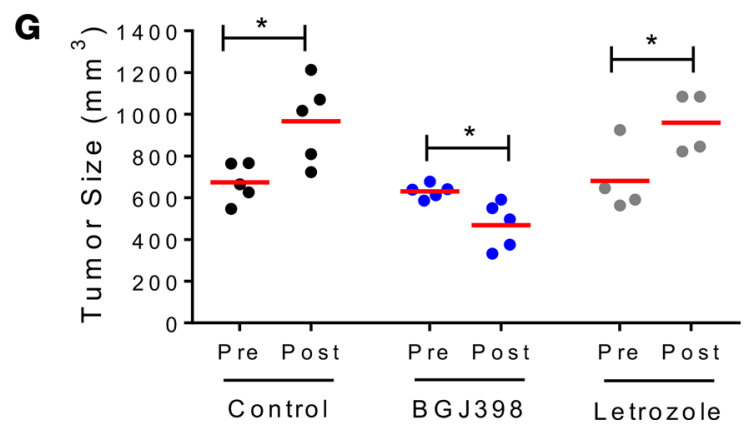

Figure 5. Link between pFGFR1 and obesity in ER-positive breast cancer. (A) Representative images of pFGFR1 staining in UCD12 PDX tumors from lean (left) or obese (right) mice after EWD. Scale bar: $100 \mu \mathrm{m}$. (B) Quantification of phospho- and total FGFR1 staining. $n=10$ each. ( ${ }^{*} P<0.05, t$ test). (C) Representative images of pFGFR1 in xenograft tumors derived from a UCD12 cell line in lean (left) or obese (right) mice after EWD. Scale bar: $70 \mu \mathrm{m}$ (D) Quantification of phospho- and total FGFR1. ( ${ }^{* *} P<0.01, t$ test). $n=7$ lean, 9 obese. (E) Representative images of pFGFR1 in primary human breast tumors collected after 4 months of neoadjuvant exemestane treatment from postmenopausal lean (left) or obese (right) women. Scale bar: $100 \mu \mathrm{m}$. (F) Quantification of phospho- and total FGFR1. $n=9, \mathrm{BMI}<25 \mathrm{~kg} / \mathrm{m}^{2} ; n=17, \mathrm{BMI} \geq 25 \mathrm{~kg} / \mathrm{m}^{2}$. ( ${ }^{*} P<0.05$, Mann Whitney U test). (G) Tumor burden in HFHS mice before (pre) and after (post) EWD, treated with vehicle (control, black); BG)398 (blue); or letrozole (gray). * $P<0.05$, paired 2 tailed $t$ test. 
A

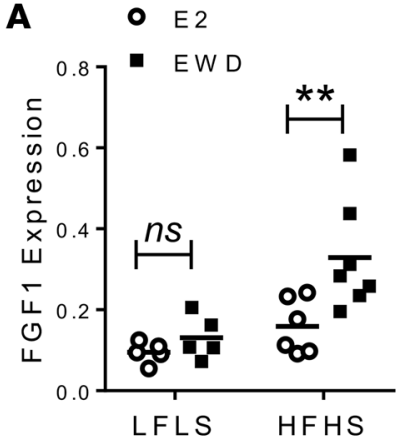

D

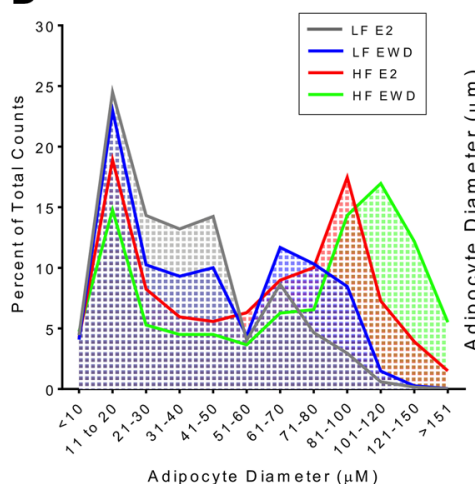

F

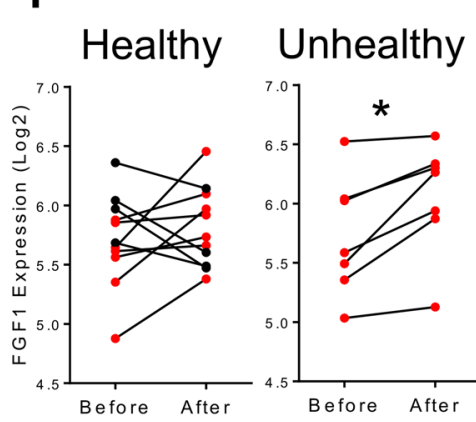

B
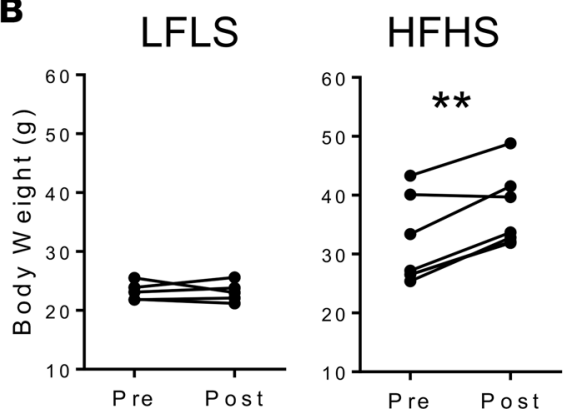

C

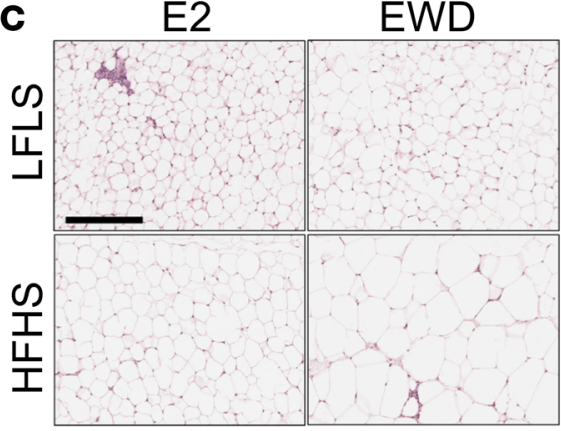

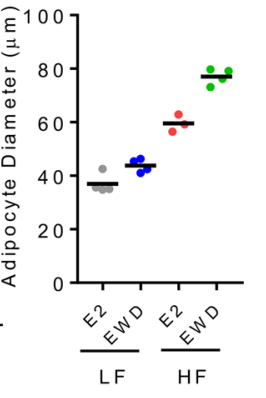

G

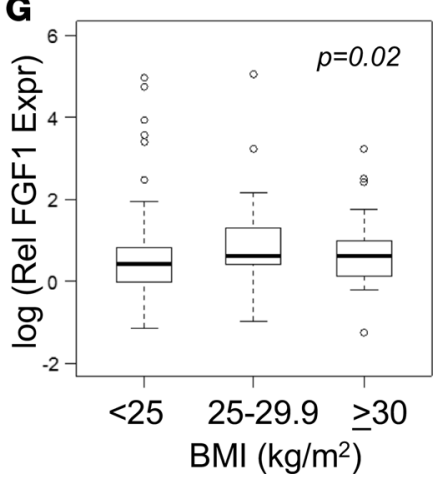

E

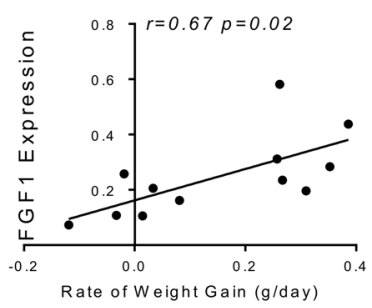

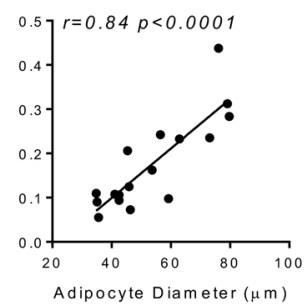

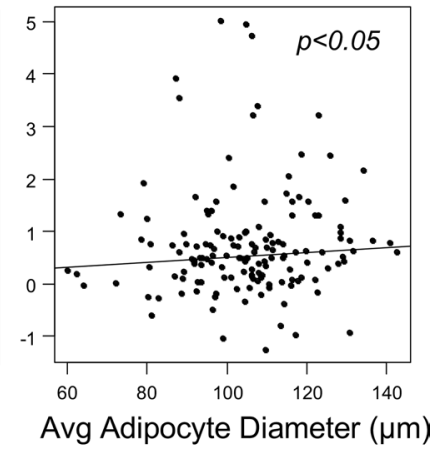

Figure 6. Weight gain in the obese promotes FGF1 expression. (A) FGF1 expression normalized to Pol2ra in mammary adipose from lean ( $n=5$ per treatment) or obese ( $n=6$ per treatment) mice with E2 or after EWD. ( ${ }^{* *} P<0.01$, unpaired $t$ test). (B) Body weigh of lean and obese mice before (pre) and 3 weeks after (post) EWD. ( ${ }^{* *} P<0.01$, paired $t$ test). (C) Representative images of mammary adipose tissue from lean or obese mice with and without supplemental E2. Scale bar: $200 \mu \mathrm{m}$. (D) Adipocyte size distribution and average adipocyte diameter per mouse shown in right panel; $>500$ cells were analyzed for at least 3 mice per group (right panel, adiposity effect, $P<0.0001$; E2 effect, $P<0.0001$; Interaction, $P=0.007,2$-way ANOVA). (E) Spearman correlation of FGF1 expression in mouse mammary adipose after EWD with rate of EWD-induced weight gain (left), mammary adipose mass (middle), and adipocyte diameter (right). (F) FGF1 expression in s.c. adipose from metabolically healthy (left) and unhealthy (right) obese subjects before and after intentional weight gain. ${ }^{*} P<0.05$, paired $t$ test. Samples in red were higher after weight gain than before. (G) Log-transformed FGF1 relative expression by BMI (left), scatter plots of BMI vs. log-transformed FGF1 relative expression (middle), and average adipocyte diameter (right) vs. log-transformed FGF1 relative expression. Tukey line, a robust linear fit of the association, is also plotted.

One innovative aspect of this study is the use of a potentially novel diet-induced obese graft-competent model to investigate the link between obesity and cancer. The breast cancer field has been limited by a lack of adequate preclinical models that represent the variety of tumor subtypes diagnosed in humans. We have overcome this limitation by grafting human cell lines and PDX tumors into mice that are susceptible to diet-induced obesity, which has allowed us to identify tumor intrinsic and extrinsic factors that likely support growth in the obese. While we attempted to promote obesity in commonly used NSG hosts, only a small percentage of adult females gained excess body fat, compared with the Rag1-null mice on the B6 background. While both models are immune compromised, the Rag1-null females retain DCs, macrophages, and NKs, which could predispose them to diet-induced obesity development. The present study 


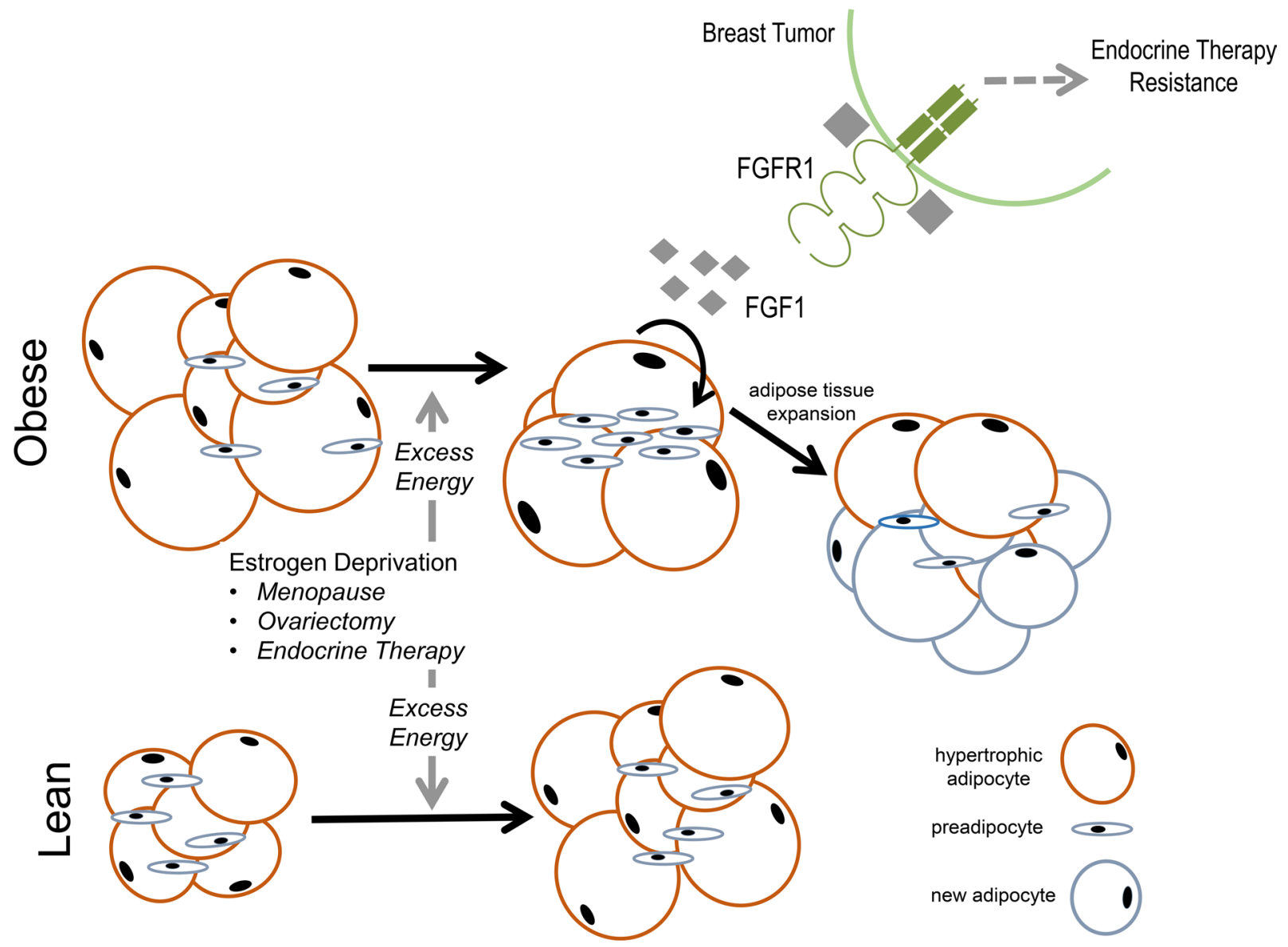

Figure 7. Working model. Obesity and excess energy activate FGFR1 in breast tumors through FGF1 produced by hypertrophic adipocytes during adipose tissue expansion. FGFR1 activation can drive endocrine therapy resistance.

has centered on FGF signaling as an example; however, it is likely that a number of factors contribute to tumor progression in this context, including excess circulating nutrients, inflammatory cytokines that alter steroid hormone signaling, and the production of other growth factors. Our observation that FGFR1 signaling associated with changes in ECM structural and remodeling proteins is consistent with its role in tumor progression and suggests the diet-induced obesity xenograft (DIOX) model could be useful to study prometastatic changes in the tumor microenvironment linked to obesity. The methods to generate DIOX mice can be applied to any cancer type that is promoted by excess adiposity and metabolic disease, including endometrial, esophageal, and colon $(48,49)$.

This powerful tool has the limitation of lacking an adaptive immune system (i.e., mature $\mathrm{B}$ and $\mathrm{T}$ cells); however, this does not preclude its use for investigating the obesity cancer relationship. Other obesity-specific variables are known to support breast cancer, including leptin, insulin/IGF1, and increased available nutrients such as glucose and fatty acids, all of which can be evaluated in immune-compromised mice. Consistent with these other pathways, IGF1R emerged as another putative driver of obesity-associated and therapy-resistant breast cancer in patient samples (Figure 1). Rag1-null mice are reported to retain functional macrophages that polarize to a proinflammatory phenotype in the obese (50), which also occurs in women (51). Notably, we identified telltale crown-like structures (CLS) in the mammary adipose tissue of HFHS-fed mice after EWD (Supplemental Figure 5), and others have reported elevated IL-6 production in Rag1-null mice after high-fat feeding (50), offering the potential to study the role of innate immunity. Currently, transplant-competent models with humanized immune systems are in their infancy but have led to important clinical advances (52). Future studies will apply methods to overcome the inherent obesity resistance displayed by many immune-compromised strains in combination with human immune cells to create a model from which to study the interaction between these 2 important variables and their influence on cancer progression. 
Another critical finding is the identification of pathways associated with ER-positive tumor promotion in the context of obesity, despite estrogen deprivation - namely, FGFR1 signaling. Inflamed adipose tissue is known to produce estrogen through elevated aromatase production by stromal cells $(51,53,54)$. While this estrogen-dependent scenario is likely to contribute to the increased incidence of ER-positive disease in women with obesity, it is unclear whether elevated estrogen contributes to poor outcomes after treatment. Although we were unable to detect E2 in mammary adipose tissue of obese Rag1-null mice, others have shown increased aromatase expression and activity, particularly in visceral adipose depots, of athymic nude mice (55). Human breast cancer cells that overexpressed aromatase were uniquely sensitive to the AI letrozole, suggesting obesity-specific regulation of aromatase function (55). Our observation that excess adiposity promoted tumor progression despite undetectable E2 levels indicates the importance of utilizing graft-competent obese models in combination with a variety of PDX tumors to identify novel mediators of endocrine therapy resistance. The benefit of such a system is that it can be combined with human tumors that express and retain ER throughout progression to metastatic disease, providing an opportunity to investigate the most prevalent breast cancer subtype.

In summary, we provide evidence that the FGF1/FGFR1 axis is an important mediator of obesity-associated growth of ER-positive tumors after estrogen deprivation by leveraging patient data and a potentially novel graft-competent diet-induced obese model. Partnering this model with human tumors, as we have done here for breast cancer, can be a powerful tool to identify intrinsic (tumor specific) and extrinsic (host specific) factors involved in the obesity-cancer relationship, and it can lead to the discovery of novel therapeutic targets.

\section{Methods}

\section{The DIOX Model}

Housing and diet. Juvenile (6 weeks old) female Rag1-null mice were purchased from The Jackson Laboratory (stock no. 002216). Upon arrival, mice were housed under standard vivarium conditions, including a 12-hour light dark cycle, and a room temperature of approximately $23^{\circ} \mathrm{C}$. Mice were placed on either a HFHS diet (Research Diets, D15031601), which contained 40\% kcal fat from butter and sucrose (292.5 gram \%) or aLFLS diet (Research Diets, D11092101), which contained $11.5 \%$ kcal fat from butter and no sucrose. Mature females (12 weeks old) were then moved to static cages that were placed on warming blankets attached to warm water pumps (Stryker, Thermo Fisher Scientific). Pumps provided continuous heat set at $40^{\circ} \mathrm{C}$. Cage and blanket temperatures were monitored with an infrared digital thermometer 3 times per day for 3 consecutive days. Control cage temperatures housed at standard room temperature were also monitored. Body weights were monitored weekly, and body composition was measured several times throughout each study using quantitative magnetic resonance (qMR; Echo MRI Whole Body Composition Analyzer, Echo Medical Systems). A similar approach was taken with NSG mice (The Jackson Laboratory, stock no. 005557), except that tumors and E2 pellets were not administered (described in detail below).

Ovariectomy and tumor studies. Adult female Rag1-null mice were OVX under isofluorane anesthesia through dorsal incisions. At the time of OVX, mice were implanted with a s.c. E2 pellet, which was a silastic tube containing cellulose and either $1 \mathrm{mg}$ or $0.25 \mathrm{mg}$ of E2 (MilliporeSigma). Mice were also grafted with either $1 \times 10^{6}$ PT12 (a cell line derived from the UCD12 PDX) or MCF7 cells, injected with an insulin syringe, or with a $2 \times 3 \mathrm{~mm}$ fragment of the UCD12 PDX tumor, implanted with a 10G trochar (Innovative Research of America). Tumors were injected/implanted into the inguinal (\#4) mammary fat pads, and tumors were measured in 2 dimensions using digital calipers. Volume was calculated as (length ${ }^{2}$ $\times$ width) $/ 2$, where the length was the longer of the 2 measurements. After tumors grew to a specific size, EWD treatment was administered by removing the silastic E2 pellet under isofluorane anesthesia. Control mice were anesthetized, and sham surgery was performed. Mice were randomized to treatment groups based on body fat percentage within adiposity groups and on tumor burden. Studies were terminated 3 weeks after EWD treatment. In a separate study, juvenile female mice were matured on HFHS diet (Research Diets, D15031601), OVX as described above, and implanted with UCD12 PDX tumors. When tumors reached approximately $650 \mathrm{~mm}^{3}$, mice were randomized based on tumor volume to EWD (control; $n=5$ mice), FGFR inhibitor (BGJ398; $10 \mathrm{mg} / \mathrm{kg}$ body weight, daily oral administration; $n=5 \mathrm{mice}$ ), or AI (letrozole, $10 \mu \mathrm{g} /$ day s.c.; $n=4$ mice) (56). Treatment continued for 3 weeks. 
Study end and tissue collection. Mice were fasted for 4-6 hours, and final body weights and body composition were collected prior to sacrifice. Blood was collected from a cardiac draw, and serum was separated and stored at $-80^{\circ} \mathrm{C}$. Tissues, including tumors, mammary glands (inguinal and thoracic), abdominal and visceral fat pads, liver, kidney, and gastrocnemius were dissected, weighed, and fixed in formalin and/ or snap frozen. Serum glucose was analyzed using a colorimetric assay (Thermo Fisher Scientific), and insulin was analyzed using an ultrasensitive ELISA (Alpco). HOMA-IR was calculated as follows: glucose $(\mathrm{mmol} / 1) \times$ insulin $(\mathrm{mU} / 1) / 22.5$.

IHC

Tissues were fixed in 10\% neutral-buffered formalin and processed according to standard histologic procedures. IHC was performed on paraffin-embedded tissue sections using antibodies against ER (RM9101; Thermo Fisher Scientific), PR (A0098; Dako North America); Ki67 (clone MIB-1; M7240; Dako North America); pFGFR1 (ab59194; Abcam), and total FGFR1 (9740; Cell Signaling Technology). Blocking and secondary antibody reagents were purchased from Vector Laboratories. Stained sections were digitally archived and quantified using the Aperio Digital Pathology System and Image Scope software (Leica Biosystems). Antibody specificity was validated in a series of breast cancer PDX tumors that varied in expression of FGFR1 (high, medium, low) and in breast cancer cell lines (PT12, in which FGFR1 was reduced using siRNA; 4390824; Ambion).

\section{Primary human breast tumor analysis}

Analysis of pFGFR1 and patient outcomes. Forty-six patients with ER-positive tumors, accrued between 1988 and 1993 at the Massachusetts General Hospital (Boston, Massachusetts, USA), who were treated with tamoxifen as part of their clinical management were used for this study. These patients were originally covered by an approved IRB protocol from the Massachusetts General Hospital and more recently by an exempt protocol from the Colorado Multiple Institutional Review Board. All studies were truncated at the end of 1998, when the last follow-up was obtained. A manual receiver operating characteristic (ROC) analysis was performed for pFGFR1 to establish a cut point that best separated those patients who failed from those who did not fail based on $\chi^{2}$ number. High pFGFR1 $(\geq 75 \%)$ was correlated with poor disease-free and breast-specific overall survival in both univariate and multivariate analyses. Stat View 5.1 (Abacus) software was used for statistical analyses of clinical data.

Analysis of pFGFR1 by BMI. The patient characteristics were previously described $(57,58)$. Briefly, the trial included postmenopausal women with locally advanced, hormone receptor-positive breast cancers treated with exemestane alone or with exemestane plus tamoxifen. Only patients treated with exemestane were included in the current analysis.

\section{Serum assays}

Serum hormone/metabolites were analyzed with the following reagents: E2 was analyzed using the Mouse/Rat Estradiol ELISA (ES180S-100; Calbiotech), insulin was analyzed using the Mouse Ultrasensitive Insulin ELISA (80-INSMSU-E01; Alpco), and glucose was analyzed using the Infinity Glucose Hexokinase Reagent (TR15421; Thermo Fisher Scientific).

\section{Tissue mass spectrometry}

Mouse and human adipose tissue were processed, and E2 was analyzed by LC-MS/MS according to our previously described methods (38).

\section{RNA sequencing}

RNA sequencing (RNA-seq) libraries were prepared from total RNA by the University of Colorado Core using the Illumina TruSeq Stranded mRNA LT Sample Prep Kit. Resulting libraries were sequenced on an Illumina HiSeq $2500(1 \times 125 \mathrm{bp})$. After demultiplexing, the resulting reads were trimmed with cutadapt (59) to remove 3 ' adaptor sequences and low quality 3 ' bases $(q<10)$. The trimmed reads were then aligned to both the human (GRCh38) and mouse (mm10) genomes using hisat2 (60). Reads were then assigned to either the human or mouse genome using disambiguate (61), and ambiguous reads were discarded. Unambiguous reads were assigned to features using Rsubread's featureCounts (62). Differential gene expression testing was performed using limma (63) and voom (64) with a design formula that included terms to control 
for donor animal identity and serum estrogen concentration. Differential expression data were visualized using degust (https://github.com/drpowell/degust). Data can be accessed through the NCBI GEO using GSE110644 (https://www.ncbi.nlm.nih.gov/geo/query/acc.cgi?acc=GSE110644).

Publicly available microarray data were processed using affy (65) and limma (63). Genes were selected for analysis in Ingenuity based on $\log _{2}$ fold change $( \pm 0.5)$. Ingenuity upstream regulator and diseases/function analyses were further interpreted. Within each of those categories, putatively activated pathways were selected by $\mathrm{z}$ score $(>0.1)$ and $P$ value $(<0.05)$. Venn diagrams were created to compare unique and shared activating pathways across gene signatures from post-letrozole treatment tumors and primary untreated ER-positive tumors from peri- and postmenopausal patients with BMI $\geq$ $25 \mathrm{~kg} / \mathrm{m}^{2}$. Datasets are available in the NCBI Gene Expression Omnibus (GEO) repository: letrozole treatment, GSE5462 (17, 18); primary tumors with BMI, GSE24185 (16).

\section{Human adipose tissue analysis}

Study design. Women who were undergoing mastectomy for breast cancer risk reduction or treatment at MSKCC were eligible. Height and weight were recorded on the day of surgery and used to calculate BMI.

Data and tissue collection. Clinicopathological data were abstracted from the electronic medical records and quality assured through independent data review. Tumor subtype was classified as ER positive and/or $\mathrm{PR}$ positive if $>1 \%$ staining by IHC was reported. HER2 was categorized as positive if IHC intensity was 3 or greater or if FISH-amplification $\geq 2$.0-fold.

For each subject, paraffin blocks and snap-frozen samples were prepared from breast tissue not involved by tumor on the day of mastectomy. Frozen samples were stored in the presence of RNALater (Ambion) at $-80^{\circ} \mathrm{C}$.

Assessment of breast adipocyte diameters. Adipocyte diameter measurements were performed as previously described (51). Briefly, nontumorous breast H\&E sections were photographed at 20× using an Olympus BX50 microscope and MicroFire digital camera (Optronics). Mean diameters were then calculated from the digitized images by measuring 30 or more individual adipocytes for each patient using the linear dimensional tool in the Canvas 11 Software (ACD Systems International Inc.).

Quantitative PCR. Total RNA was isolated from human breast tissue using the RNeasy Mini Kit (Qiagen). RNA (2,000 ng) was reverse transcribed using the qScript cDNA Synthesis Kit (QuantaBio), and the resulting CDNA used for real-time PCR amplification with Fast SYBR green PCR master mix on a 7500 HT real-time PCR system (Applied Biosystems). GAPDH was used as an endogenous normalization control. Primers for GAPDH (QT00079247) and FGF1 (QT00079317) were purchased from Qiagen. Relative levels of FGF1 were determined using the $\triangle \triangle \mathrm{CT}$ analysis protocol.

\section{Statistics}

Shapiro-Wilk normality test was used; if data did not pass $(P<0.05)$, non parametric analyses were used. For each figure, the statistical test used and significance value are reported in the legend. All $t$ tests were 2-tailed. A $P$ value less than 0.05 was considered significant. Patient clinicopathologic features were summarized in terms of median and interquartile range (IQR) for continuous variables and for counts and percentages for categorical variables. The association between the expression levels of FGF1 and BMI and the association between FGF1 expression and average adipocyte diameters were examined using the linear mixed-effects model to account for potential differences in sample cohorts and experimental plates. Levels of FGF1 expression were log transformed to ensure the underlying model assumptions are satisfied. Statistical analyses were carried out using $\mathrm{R}$ version 3.3.1.

\section{Study approval}

The studies in humans were reviewed and approved by appropriate IRBs at either the University of Colorado, MSKCC, and/or Weill Cornell Medical College, as applicable. Informed consent was obtained from subjects prior to participation in any study. The studies conducted in animals were reviewed and approved by the University of Colorado IACUC.

\section{Author contributions}

All authors provided substantial contributions to the concept and design of the studies and manuscript. Conception and design were contributed by EAW, PSM, PK, and CAS. Development of methods was contributed by EAW, AEG, SJJ, MCR, SME, XKZ, and AJD. Acquisition of data (provided animals, acquired 
and managed patients, provided facilities, etc.) was contributed by EAW, BMJ, ADT, SMA, AE, NMI, MM, DJF, OE, AJD, CAS, and PK. Analysis and interpretation of data were contributed by EAW, PK, AEG, BMJ, HMB, SME, ADT, XKZ, AJD, CAS, and PSM. Administrative, technical, or material support were contributed by AEG, SJJ, SME, and XKZ.

\section{Acknowledgments}

This research was supported by the Komen Foundation (CCR17483321 to EAW), the NIH (KL2 CTSA UL1TR002534 to EAW; NCI R01CA205044 to PK; NCI R01 CA215797 and NCI U54 CA210184 to AJD; NCI CA164166 and NICHD P50 HD073063 to PSM; NCI R01CA140985 to CAS; NIDDK P30DK048520 Nutrition and Obesity Research Center awards to EAW and PSM; and P30 CA046934 to the University of Colorado Cancer Center), the Breast Cancer Research Foundation (to CAS, PK, and AJD), the Botwinick-Wolfensohn Foundation (in memory of Mr. and Mrs. Benjamin Botwinick; to AJD), the Cancer League of Colorado, and the University of Colorado Department of Medicine Team Science Award.

Address correspondence to: Elizabeth A. Wellberg, University of Colorado Anschutz Medical Campus, Department of Pathology, Mail Stop 8104, Aurora, Colorado 80045, USA. Phone: 303.724.3471; Email: elizabeth.wellberg@ucdenver.edu.

1. Sahin S, et al. The association between body mass index and immunohistochemical subtypes in breast cancer. Breast. 2017;32:227-236

2. Bonsang-Kitzis H, et al. Beyond Axillary Lymph Node Metastasis, BMI and Menopausal Status Are Prognostic Determinants for Triple-Negative Breast Cancer Treated by Neoadjuvant Chemotherapy. PLoS ONE. 2015;10(12):e0144359.

3. Protani M, Coory M, Martin JH. Effect of obesity on survival of women with breast cancer: systematic review and meta-analysis. Breast Cancer Res Treat. 2010;123(3):627-635.

4. Ligibel JA, et al. Body Mass Index, PAM50 Subtype, and Outcomes in Node-Positive Breast Cancer: CALGB 9741 (Alliance) J Natl Cancer Inst. 2015;107(9):djv179.

5. Jiralerspong S, Kim ES, Dong W, Feng L, Hortobagyi GN, Giordano SH. Obesity, diabetes, and survival outcomes in a large cohort of early-stage breast cancer patients. Ann Oncol. 2013;24(10):2506-2514.

6. Cleary MP, Grossmann ME. Minireview: Obesity and breast cancer: the estrogen connection. Endocrinology. 2009;150(6):2537-2542.

7. Burstein HJ, et al. Adjuvant endocrine therapy for women with hormone receptor-positive breast cancer: american society of clinical oncology clinical practice guideline focused update. J Clin Oncol. 2014;32(21):2255-2269.

8. Folkerd EJ, Dixon JM, Renshaw L, A'Hern RP, Dowsett M. Suppression of plasma estrogen levels by letrozole and anastrozole is related to body mass index in patients with breast cancer. J Clin Oncol. 2012;30(24):2977-2980.

9. Folkerd EJ, Dixon JM, Renshaw L, A'Hern RP, Dowsett M. Suppression of plasma estrogen levels by letrozole and anastrozole is related to body mass index in patients with breast cancer. J Clin Oncol. 2012;30(24):2977-2980.

10. Sestak I, Distler W, Forbes JF, Dowsett M, Howell A, Cuzick J. Effect of body mass index on recurrences in tamoxifen and anastrozole treated women: an exploratory analysis from the ATAC trial. J Clin Oncol. 2010;28(21):3411-3415.

11. Murphy CG, Dickler MN. Endocrine resistance in hormone-responsive breast cancer: mechanisms and therapeutic strategies. Endocr Relat Cancer. 2016;23(8):R337-R352.

12. Ogden CL, Carroll MD, Kit BK, Flegal KM. Prevalence of childhood and adult obesity in the United States, 2011-2012. JAMA. 2014;311(8):806-814.

13. Meehan TF, et al. PDX-MI: Minimal Information for Patient-Derived Tumor Xenograft Models. Cancer Res. 2017;77(21):e62-e66.

14. Formisano L, et al. Association of FGFR1 with ER $\alpha$ Maintains Ligand-Independent ER Transcription and Mediates Resistance to Estrogen Deprivation in ER+ Breast Cancer. Clin Cancer Res. 2017;23(20):6138-6150.

15. Turner N, et al. FGFR1 amplification drives endocrine therapy resistance and is a therapeutic target in breast cancer. Cancer Res. 2010;70(5):2085-2094.

16. Creighton CJ, et al. A gene transcription signature of obesity in breast cancer. Breast Cancer Res Treat. 2012;132(3):993-1000.

17. Miller WR, Larionov A. Changes in expression of oestrogen regulated and proliferation genes with neoadjuvant treatment high light heterogeneity of clinical resistance to the aromatase inhibitor, letrozole. Breast Cancer Res. 2010;12(4):R52.

18. Miller WR, et al. Changes in breast cancer transcriptional profiles after treatment with the aromatase inhibitor, letrozole. Phar macogenet Genomics. 2007;17(10):813-826.

19. Fan C, et al. Concordance among gene-expression-based predictors for breast cancer. N Engl J Med. 2006;355(6):560-569.

20. Wirapati P, et al. Meta-analysis of gene expression profiles in breast cancer: toward a unified understanding of breast cancer subtyping and prognosis signatures. Breast Cancer Res. 2008;10(4):R65

21. Fagan DH, Yee D. Crosstalk between IGF1R and estrogen receptor signaling in breast cancer. J Mammary Gland Biol Neoplasia. 2008;13(4):423-429

22. Black BL, Croom J, Eisen EJ, Petro AE, Edwards CL, Surwit RS. Differential effects of fat and sucrose on body composition in A/J and C57BL/6 mice. Metab Clin Exp. 1998;47(11):1354-1359.

23. Surwit RS, et al. Differential effects of fat and sucrose on the development of obesity and diabetes in C57BL/6J and A/J mice. Metab Clin Exp. 1995;44(5):645-651.

24. Mombaerts P, Iacomini J, Johnson RS, Herrup K, Tonegawa S, Papaioannou VE. RAG-1-deficient mice have no mature B and T lymphocytes. Cell. 1992;68(5):869-877. 
25. Stemmer K, et al. Thermoneutral housing is a critical factor for immune function and diet-induced obesity in C57BL/6 nude mice. Int J Obes (Lond). 2015;39(5):791-797.

26. Speakman JR, Keijer J. Not so hot: Optimal housing temperatures for mice to mimic the thermal environment of humans. Mol Metab. 2012;2(1):5-9.

27. Fleming TP, et al. Origins of lifetime health around the time of conception: causes and consequences. Lancet. 2018;391(10132):1842-1852.

28. McMullen S, Mostyn A. Animal models for the study of the developmental origins of health and disease. Proc Nutr Soc. 2009;68(3):306-320

29. Sinclair KD, Lea RG, Rees WD, Young LE. The developmental origins of health and disease: current theories and epigenetic mechanisms. Soc Reprod Fertil Suppl. 2007;64:425-443.

30. Matthews SB, Sartorius CA. Steroid Hormone Receptor Positive Breast Cancer Patient-Derived Xenografts. Horm Cancer. 2017;8(1):4-15

31. Eliassen AH, et al. Endogenous steroid hormone concentrations and risk of breast cancer among premenopausal women. $J$ Natl Cancer Inst. 2006;98(19):1406-1415.

32. Nishino M, Hayakawa K, Nakamura Y, Morimoto T, Mukaihara S. Effects of tamoxifen on hepatic fat content and the development of hepatic steatosis in patients with breast cancer: high frequency of involvement and rapid reversal after completion of tamoxifen therapy. AJR Am J Roentgenol. 2003;180(1):129-134.

33. Sheean PM, Hoskins K, Stolley M. Body composition changes in females treated for breast cancer: a review of the evidence. Breast Cancer Res Treat. 2012;135(3):663-680.

34. Mauvais-Jarvis F, Clegg DJ, Hevener AL. The role of estrogens in control of energy balance and glucose homeostasis. Endocr Rev. 2013;34(3):309-338.

35. Mayes JS, Watson GH. Direct effects of sex steroid hormones on adipose tissues and obesity. Obes Rev. 2004;5(4):197-216.

36. Checkley LA, et al. Metformin Accumulation Correlates with Organic Cation Transporter 2 Protein Expression and Predicts Mammary Tumor Regression In Vivo. Cancer Prev Res (Phila). 2017;10(3):198-207.

37. Giles ED, Jackman MR, MacLean PS. Modeling Diet-Induced Obesity with Obesity-Prone Rats: Implications for Studies in Females. Front Nutr. 2016;3:50.

38. Wellberg EA, et al. The Androgen Receptor Supports Tumor Progression After the Loss of Ovarian Function in a Preclinical Model of Obesity and Breast Cancer. Horm Cancer. 2017;8(5-6):269-285.

39. Kabos P, et al. Patient-derived luminal breast cancer xenografts retain hormone receptor heterogeneity and help define unique estrogen-dependent gene signatures. Breast Cancer Res Treat. 2012;135(2):415-432.

40. Sikora MJ, et al. Invasive lobular carcinoma cell lines are characterized by unique estrogen-mediated gene expression patterns and altered tamoxifen response. Cancer Res. 2014;74(5):1463-1474

41. Seo BR, et al. Obesity-dependent changes in interstitial ECM mechanics promote breast tumorigenesis. Sci Transl Med. 2015;7(301):301ra130.

42. Holdman XB, et al. Upregulation of EGFR signaling is correlated with tumor stroma remodeling and tumor recurrence in FGFR1-driven breast cancer. Breast Cancer Res. 2015;17:141.

43. Hutley LJ, et al. A putative role for endogenous FGF-2 in FGF-1 mediated differentiation of human preadipocytes. Mol Cell Endocrinol. 2011;339(1-2):165-171.

44. Jonker JW, et al. A PPAR $\gamma$-FGF1 axis is required for adaptive adipose remodelling and metabolic homeostasis. Nature. 2012;485(7398):391-394.

45. Fabbrini E, et al. Metabolically normal obese people are protected from adverse effects following weight gain. J Clin Invest. 2015;125(2):787-795.

46. André F, et al. Targeting FGFR with dovitinib (TKI258): preclinical and clinical data in breast cancer. Clin Cancer Res. 2013;19(13):3693-3702.

47. Giles ED, et al. Obesity and overfeeding affecting both tumor and systemic metabolism activates the progesterone receptor to contribute to postmenopausal breast cancer. Cancer Res. 2012;72(24):6490-6501.

48. Basen-Engquist K, Chang M. Obesity and cancer risk: recent review and evidence. Curr Oncol Rep. 2011;13(1):71-76.

49. Keum N, et al. Adult weight gain and adiposity-related cancers: a dose-response meta-analysis of prospective observational studies. J Natl Cancer Inst. 2015;107:djv088.

50. Lee YS, et al. Inflammation is necessary for long-term but not short-term high-fat diet-induced insulin resistance. Diabetes. 2011;60(10):2474-2483

51. Morris PG, et al. Inflammation and increased aromatase expression occur in the breast tissue of obese women with breast cancer. Cancer Prev Res (Phila). 2011;4(7):1021-1029.

52. Shultz LD, Brehm MA, Garcia-Martinez JV, Greiner DL. Humanized mice for immune system investigation: progress, promise and challenges. Nat Rev Immunol. 2012;12(11):786-798

53. Iyengar NM, Gucalp A, Dannenberg AJ, Hudis CA. Obesity and Cancer Mechanisms: Tumor Microenvironment and Inflammation. J Clin Oncol. 2016;34(35):4270-4276.

54. Subbaramaiah K, et al. Increased levels of COX-2 and prostaglandin E2 contribute to elevated aromatase expression in inflamed breast tissue of obese women. Cancer Discov. 2012;2(4):356-365.

55. Schech A, Yu S, Goloubeva O, McLenithan J, Sabnis G. A nude mouse model of obesity to study the mechanisms of resistance to aromatase inhibitors. Endocr Relat Cancer. 2015;22(4):645-656.

56. Sartorius CA, et al. Estrogen promotes the brain metastatic colonization of triple negative breast cancer cells via an astrocyte-mediated paracrine mechanism. Oncogene. 2016;35(22):2881-2892.

57. Harvell DM, et al. Estrogen regulated gene expression in response to neoadjuvant endocrine therapy of breast cancers: tamoxifen agonist effects dominate in the presence of an aromatase inhibitor. Breast Cancer Res Treat. 2008;112(3):489-501.

58. Harvell DM, et al. Molecular signatures of neoadjuvant endocrine therapy for breast cancer: characteristics of response or intrinsic resistance. Breast Cancer Res Treat. 2008;112(3):475-488.

59. Martin M. Cutadapt removes adapter sequences from high-throughput sequencing reads. EMBnetjournal. 2011;17(1):10-12. 
60. Kim D, Langmead B, Salzberg SL. HISAT: a fast spliced aligner with low memory requirements. Nat Methods. 2015;12(4):357-360.

61. Ahdesmäki MJ, Gray SR, Johnson JH, Lai Z. Disambiguate: An open-source application for disambiguating two species in next generation sequencing data from grafted samples. F1000Res. 2016;5:2741.

62. Liao Y, Smyth GK, Shi W. featureCounts: an efficient general purpose program for assigning sequence reads to genomic features. Bioinformatics. 2014;30(7):923-930.

63. Ritchie ME, et al. limma powers differential expression analyses for RNA-sequencing and microarray studies. Nucleic Acids Res. 2015;43(7):e47.

64. Law CW, Chen Y, Shi W, Smyth GK. voom: Precision weights unlock linear model analysis tools for RNA-seq read counts. Genome Biol. 2014;15(2):R29.

65. Gautier L, Cope L, Bolstad BM, Irizarry RA. affy--analysis of Affymetrix GeneChip data at the probe level. Bioinformatics. 2004;20(3):307-315 